\title{
Síndromede Moebius. A propósito de un caso.
}

'Médico Cirujano. Departamento de Ciencias Morfológicas. Escuela de Ciencias de la Salud, Dr. "Francisco Battistini Casalta", Universidad De Oriente. Ciudad Bolívar. Venezuela.

${ }^{2}$ Médico Pediatra. Servicio de Emergencia Pediátrica. Complejo Hospitalario Universitario Ruiz y Páez. Ciudad Bolívar. Venezuela.

${ }^{2}$ Neurólogo Pediátrico. Servicio de Neurología Pediátrica. Complejo Hospitalario Universitario Ruiz y Páez. Ciudad Bolívar. Venezuela.

Correspondencia a: Luis Alfredo Hernández Villarroel

luisvango@hotmail.com

Palabras clave: Síndrome de Moebius, anomalías congénitas, nervios craneales, parálisis facial.

Keywords: Moebius syndrome, congenital
Procedencia y arbitraje: no comisionado, sometido a arbitraje externo.

Recibido para publicación: 28 de Septiembre del 2016 Aceptado para publicación 09 de Diciembre del 2016

Citar como:

Rev Cient Cienc Med

2016; 19(2): 60-64

\author{
Moebius Syndrome: A Case Report \\ Hernández Villarroel Luis Alfredo1, Lanz Luna Jehan Lym¹, Zavala Valladares José2, Machuca \\ Carolina $^{3}$
}

\section{RESUMEN}

Anomalía congénita caracterizada por afectación del VI y VII par craneano que determina una parálisis facial, uni o bilateral, aunque el compromiso puede ser más extenso. Se describe paciente masculino de 4 años de edad, obtenido por cesárea, con antecedentes de amenaza de aborto en cuatro oportunidades a partir de la décima semana de gestación; en el período de lactancia presentó dificultad para la succión y deglución. Al examen físico, facies inexpresiva, boca "en carpa", parálisis facial bilateral, estrabismo, nistagmos, puente nasal ancho, micronagtia, paladar hendido, pie equino varo bilateral y sindactilia en mano derecha. Se relaciona con eventos y fármacos (misoprostol) que puedan producir una disrupción vascular e insuficiencia placentaria. Se sugiere como causa, la afección hipóxico/isquémica transitoria del feto debido a alteraciones en la circulación materno-fetal durante primer trimestre de embarazo, por amenaza de aborto y probablemente exposición a teratógenos como el misoprostol.

\section{ABSTRACT}

Congenital anomaly characterized by involvement of the VI and VII cranial nerve that causes a bilateral facial paralysis, or uni, but the commitment may be longer. Is described, male patient aged 4, obtained by cesarean section with a history of threatened abortion four times from the tenth week of pregnancy; in lactancy, presented difficulty in sucking and swallowing. On physical examination, expressionless face, mouth "in tents", bilateral facial paralysis, strabismus, nystagmus, broad nasal bridge, micrognathia, cleft palate, clubfoot and bilateral syndactyly in right hand.It relates to events and drug (misoprostol) that produce vascular disruption and placental insufficiency. In this patient had been suggested as a cause transient hypoxic / ischemic disease of the fetus due to alterations in maternal-fetal circulation during early pregnancy, threatened abortion and likely exposure to teratogens such as misoprostol.

\section{INTRODUCCIÓN}

$\mathrm{E}^{1}$ Síndrome de Moebius, es una patología de origen congénito con una baja incidencia en la población, del cual, se ha realizado alrededor de 500 reportes clínicos en la literatura ${ }^{1}$. Fue descrita en un inicio por von Graefe y Möbius en 1880 y $1888^{2}$. Se le conoce también como diplejía facial congénita o agenesia nuclear ${ }^{3}$, enfermedad en la que se ven afectados los núcleos donde se originan los pares craneales VI y VII impidiendo su total desarrollo ${ }^{4}$; produciéndose en consecuencia parálisis del nervio facial uni o bilateral, acompañada de incapacidad para la abducción ocular, presente desde el nacimiento ${ }^{5}$.

En un estudio realizado en el Hospital Universitario "La Fe de Valencia” de España, se investigó la frecuencia del Síndrome Moebius, determinándose que ésta se sitúa entre 1/490.000 y 1/677.000 habitantes con una incidencia anual de un caso por cada 115.000 nacidos vivos (aproximadamente 3 o 4 casos nuevos cada año en España) ${ }^{6}$.

Éste síndrome tiene diversas formas de presenta- ción clínica, por ello se ha realizado una clasificación basada en su forma de presentación: Síndrome de Moebius clásico, caracterizado por parálisis bilateral parcial o completa de los nervios facial y motor ocular externo, el cual tiene una frecuencia de $60 \%$ de los casos presentados; y Síndrome de Moebius asociado a otras anomalías, que corresponde al $40 \%$ de los ca$\operatorname{sos}^{7}$

Algunos pacientes pueden tener un compromiso más extenso, pudiendo afectar el III, IV, V, IX, X y XII par craneal. Pueden presentar además disfagia, disfonía, dificultad para la succión, anormalidades cráneofaciales y defectos en la extremidades en aproximadamente $50 \%$ de los $\operatorname{casos}^{8,9}$.

Las anormalidades músculo-esqueléticas ocurren en un tercio de los casos y pueden comprender pie Equinovaro congénito ( $30 \%$ de los casos), sindactilia, braquidactilia, ectrodactilia, o defectos de reducción más severos en $20 \%$ de los casos. Con menos frecuencia ( $15 \%$ de los casos), se asocia a síndrome 
de Poland y ocasionalmente a anomalía de KlippelFeil $^{8,10}$.

También pueden verse defectos cardiacos, urinarios, hipogenitalismo e hipogonadismo. Puede haber retraso mental, el cual es generalmente leve y se presenta en el 10-30\% de los pacientes. Pueden presentar autismo en el $25 \%$ de los casos aproximadamente ${ }^{8}$.

El propósito de la revisión de este caso clínico, es dar a conocer las características clínicas presentadas en un paciente con Síndrome de Moebius y otras alteraciones asociadas, que afectan su calidad de vida.

\section{PRESENTACIÓN DEL CASO}

Se describe caso de paciente masculino, de 4 años de edad,que consulta a centro de salud por presentar deformidades en miembros inferiores y la mano derecha, las cuales dificultaban la marcha y funcionabilidad . Es evaluado y diagnosticado de pie equino varo bilateral y sindactilia en mano derecha; posteriormente es referido al servicio de traumatología del Complejo Hospitalario Universitario Ruíz y Páez para su resolución quirúrgica.

Historia anterior: producto de segunda gesta, embarazo no controlado, obtenido por cesárea con antecedentes de amenaza de aborto en cuatro oportunidades a partir de la décima semana de gestación; en el período de lactancia presentó dificultad para la succión y deglución. Cabe destacar, que se presume la inducción del aborto mediante el uso de misoprostol, debido a las contradicciones de la madre durante el interrogatorio de datos relacionados a amenazas de aborto.

$\mathrm{Al}$ examen físico: facies inexpresiva, boca "en carpa”, parálisis facial bilateral, estrabismo, nistagmos, puente nasal ancho, micronagtia (Tabla 1 y Figuras 1 y 2), disfagia, disartria, paladar hendido, pie equino varo bilateral y sindactilia en mano derecha. Por lo cual se decide su valoración por neurología pediátrica, quien en conjunto con genética, realizan el diagnóstico de Síndrome de Moebius asociado a otras anomalías. Se realiza además, evaluación por cardiología quienes no encuentran otras alteraciones.

Se solicita Resonancia Magnética Cerebral y Cariotipo. Sin embargo, por ser el paciente de bajos recursos, hasta la fecha no se han podido realizar estos estudios.

Entre los Diagnósticos diferenciales se puede mencionar: parálisis facial congénita, la distrofia miotónica infantil, y/o traumática si se empleó fórceps. El diagnóstico diferencial con la parálisis facial congénita es neuropatológico, pero en esta entidad no se observan la afección de otros pares craneales. En la distrofia miotónica, existe afección de los músculos faciales la cual produce una facies inexpresiva, afec- tándose también los músculos de la deglución y de la fonación; este además clínicamente se manifiesta por la imposibilidad de relajar el músculo, manifestando la imposibilidad de abrir la mano y después cerrarla, teniendo además en un alto porcentaje manifestaciones cardiacas que pueden ser muy severas. Estos diagnósticos se descartaron dado los antecedentes prenatales del paciente y la ausencia de otros signos clínicos característicos de las entidades mencionadas.

Al sexto día de internación se realiza tenotomía percutánea bilateral del tendón de Aquiles y cura de sindactilia. Acto quirúrgico que transcurrió sin complicaciones. Es dado de alta médica al décimo día de Hospitalización y actualmente el paciente se encuentra en control por neurología y traumatología; permaneciendo con yeso inguinopédico bilateral y férula antebraquiopalmar (Figura 3).

Figura 1: Estrabismo convergente y puente nasal ancho en niño con Síndrome de Moebius.

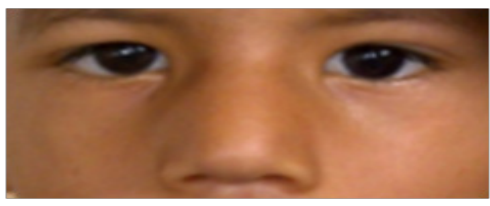

Figura 2: Boca en carpa y micrognatia en niño con Síndrome de Moebius.

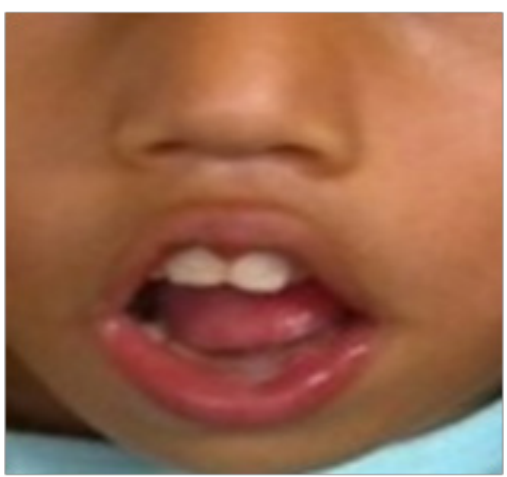

Figura 3: Yeso inguino-pédico, en paciente con Síndrome de Moebius posterior a acto quirúrgico para corrección de Pie Equinovaro bilateral.

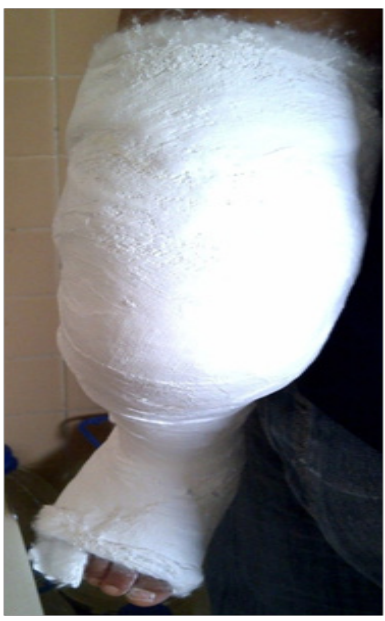


Tabla 1: Signos y síntomas presentes en paciente con Síndrome de Moebius.

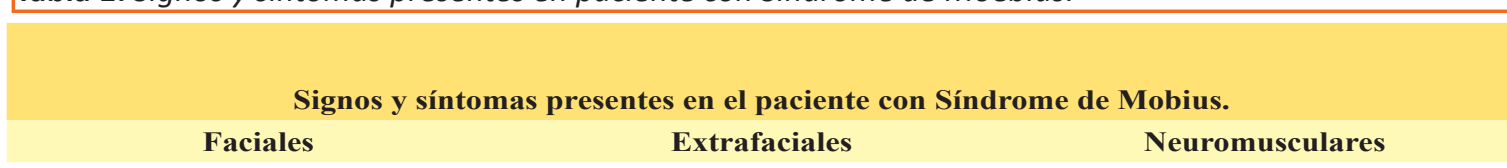

Alteración de la movilidad ocular, Pie equinovaro bilateral Trastornos de la deglución, disartria. estrabismo convergente; afección del motor ocular externo (MOE)

\begin{tabular}{|c|c|c|}
\hline Parálisis facial congénita bilateral & Sindactilia unilateral derecha. & - \\
\hline Facies inmóvil, rígida, inexpresiva & - & - \\
\hline $\begin{array}{l}\text { Otras: micronagtia, puente nasa } \\
\text { ancho, paladar hendido }\end{array}$ & - & - \\
\hline
\end{tabular}

\section{DISCUSIÓN}

De acuerdo a las conclusiones de la Conferencia Científica sobre Síndrome de Moebius realizada en Bethesda, en el año 2007, los criterios mínimos necesarios para diagnóstico de esta enfermedad son: presencia de debilidad facial no progresiva congénita unilateral o bilateral; abducción ocular limitada; y características adicionales como: compromiso de otros nervios craneales, alteraciones orofaciales, musculoesqueléticas, del neurodesarrollo o problemas sociales. Criterios de Exclusión: Ausencia de parálisis facial y/o ausencia de parálisis de nervio abducens ${ }^{11}$.

Abramson y col, clasificaron y graduaron el síndrome en base a los hallazgos clínicos encontrados, tales como parálisis de nervios craneales y anormalidades musculoesqueléticas usando el acrónimo CLUFT (nervios craneales, miembros inferiores, miembros superiores, cara y tórax $)^{12,13}$. En el caso reportado, los déficits estructurales y / o funcionales de cada componente se calificaron en una escala de 0 a 3, evidenciándose los siguientes grados: $2 / 3$ en nervios craneales, $1 / 3$ en extremidades inferiores, $1 / 3$ en extremidades superiores, $2 / 3$ con respecto a anomalías estructurales faciales y $0 / 3$ en anormalidades del tórax.

Son también características comunes: estrabismo convergente, alteraciones musculo esqueléticas como pie equinovaro, disartria y facies inexpresiva ${ }^{14,}$ las cuales también fueron evidenciadas en este paciente.

Pocos casos de este síndrome han sido descritos en la literatura radiológica ${ }^{15}$. En el caso presentado hasta el momento no ha sido posible la realización de estudios radiológicos como la resonancia magnética (RMN) y tomografía axial computarizada (TAC). No obstante, es imprescindible destacar que en estu- dios radiológicos previos en pacientes con Síndrome de Moebius, se ha identificado hipoplasia del tallo cerebral y el hallazgo universal de la completa ausencia del nervio facial. Algunos autores han concluido que estas anormalidades soportan la idea de que el Síndrome de Moebius es parte de una anomalía global de la fosa posterior ${ }^{16}$. En otros estudios de RMN se encontraron anomalías congénitas de la fosa posterior, incluyendo malformaciones de Arnold-Chiari, quiste pineal, hipoplasia de cerebelo y ventrículos asimétricos, hallazgos compatibles con defectos de desarrollo del rombencéfalo que incluye los núcleos y nervios corticoespinales o corticobulbocereberales ${ }^{17}$.

En otros estudios imagenológicos de TAC y RMN, fueron observados: depresión del 4to ventrículo, ausencia de los colículos mediales a nivel del puente, ausencia de la prominencia del hipogloso, calcificación en puente en la región del núcleo abducens. Adicionalmente, Oaunounou y col, describieron además la ausencia de pedúnculos cereberales medios en este síndrome ${ }^{12}$.

La etiología de esta patología ha sido ampliamente debatida, siendo atribuido su origen a alteraciones del desarrollo en el mesodermo o ectodermo embrionario. Igualmente, se ha descrito su asociación con teratógenos y factores hereditarios ${ }^{17}$. Sin embargo en la actualidad sólo es posible afirmar que este síndrome es la resultante de diversos factores concomitantes no bien definidos ${ }^{18,19}$.

La mayoría de los casos reportados del Síndrome de Moebius son de aparición esporádica, cuyas causas son desconocidas y a pesar de que en la literatura consultada exiten múltiples controversias en lo que a su etiología respecta, se han descrito varias teorías sobre su posible origen ${ }^{3}$. 
La isquemia fetal transitoria es la teoría descrita con mayor aceptación para explicar el origen de esta patología. Se explica, como un defecto del desarrollo secundario a un fenómeno disruptivo vascular. Según, los autores que apoyan esta teoría, una alteración que provoque cambios en el flujo sanguíneo placentario durante el principio de desarrollo del feto, puede originar daños en una extensión variable como aplasia o hipoplasia de los núcleos de nervios craneales como el nervio facial, llevándonos a evidenciar las características clínicas presentadas en esta enfermedad ${ }^{18,19}$. Se ha propuesto que estos eventos isquémicos ocurren en zonas avasculares mediales y paramediales del tronco encefálico y son secuandarias a alteraciones en el flujo de la arteria subclavia primitiva durante su desarrollo, previo al establecimiento de una irrigación adecuada a nivel del tronco encefálico por las arterias vertebrales ${ }^{11}$.

Este síndrome se produce cuando la disrupción ocurre en un preciso período del desarrollo embriológico, entre la 5ta y 8 va semana de gestación. Cualquier evento patológico que perturbe la circulación durante este período crítico causara alteraciones en el tronco cerebral o defectos generalizados del desarrollo. Pocos casos tienen ocurrencia familiar, algunas veces asociados a cambios en el cariotipo. El por qué los núcleos de los nervios craneales VI y VII son preferentemente afectados, no ha sido entendido por completo. Algunos autores, han propuesto que el embrión es particularmente vulnerable en esta etapa del desarrollo debido a la ubicación de los núcleos de los pares craneales VI y VII, localizados en la región ventral del rombencéfalo. Ubicación cercana a la plicatura, en una porción delgada, dilatada del cerebro con una relativa falta de tejido. Si la flexión es producida en esta región se provocará una disminución del flujo sanguíneo ${ }^{20}$.

El misoprostol induce intensas contracciones uterinas que pueden provocar una reducción del flujo sanguíneo, responsable de isquemia o hipoxia, que produce lesión celular endotelial y daños tisulares. Produciendo además, flexión a nivel del área de los núcleos de nervios craneales VI y VII. Del mismo modo, se puede producir afección de otros nervios craneales y otras malformaciones como parte de este Síndrome ${ }^{21}$.

\section{REFERENCIAS}

1. Ahmad M, Silvera C, Hamdan M. MOEBIUS-POLANDSYNDROME: A CASE REPORT. Salud Uninorte 2012; 28 (1): 171-7. Acceso 20 de Octubre 2015. Disponible en: http://rcientificas.uninorte.edu.co/index.php/salud/ article/viewFile/4356/2853

2. Fernández C, Hernández E, Silvera C, Jiménez B, Quin-
En Brasil, se ha repostado la asociación la administración de misoprostol durante la gestación y el Síndrome de Moebius y diferentes defectos en extremidades; sin embargo, en Venezuela, el primer reporte de esta asociación, fue realizado en el 2003 por Sánchez y col, a pesar del uso frecuente de este fármaco

En casos de presentación familiar se ha evidenciado diferentes formas de transmisión autosómicos dominantes, autosómicos recesivos y ligada al cromosoma X. En estudios, donde se ha realizado análisis citogenético en pacientes con esta patología se encontraron cariotipos normales ${ }^{6}$.

En este paciente, luego de la valoración de antecedentes prenatales y perinatales, se ha sugerido como causa la injuria hipóxico/isquémica transitoria del feto debido a alteraciones de la circulación materna, por posible insuficiencia placentaria o uso de teratógenos como el misoprostol, dado el antecedente de amenaza de aborto en el primer trimestre de embarazo.

Se han descrito también alteraciones cardiovasculares asociadas a este síndrome, tales como, defectos septales ventriculares, dextrocardia, transposición de grandes vasos6. En la evaluación cardiológica de este paciente, no se encontró ninguna alteración cardiaca asociada.

Se debe de considerar a los pacientes con Síndrome de Moebius como una patología compleja que no sólo involucra ausencia, hipoplasia o lesión destructiva de nervios craneales, sino una serie de alteraciones del desarrollo que afectan la calidad de vida del individuo. Estos pacientes deben ser controlados por un equipo multidisciplinario que incluya pediatras, neurólogos, genetistas, oftalmólogo, ortopedistas, cirujanos plásticos, ortodoncistas, otorrinolaringólogos y terapeutas del lenguaje ${ }^{5}$; de igual modo el manejo psicológico del paciente y de la familia es indispensable. Se han descrito múltiples técnicas de cirugía reconstructiva que ayudan a mejorar el aspecto y la funcionabilidad de las áreas afectadas, contribuyendo además a una mejor adaptación social del paciente. El pronóstico de esta patología depende de su asociación con otros síndromes o enfermedades; en este caso el pronóstico es bueno, debido a que no se encuentra asociado a ninguna otra entidad.

tero E,Idrovo A, et al. SÍNDROME DE MOEBIUS: GENOPATÍA VS EFECTO TERATOGÉNICO. Salud Uninorte 2006; 22 (2):182-7. Acceso 20 de Octubre 2015. Disponible en: http://rcientificas.uninorte.edu.co/index. php/salud/article/viewArticle/4096

3. Camacho R, Avila R, González L, Plascencia V, Trejo R, 
Yunes J, et al. SINDROME DE MOEBIUS. Arch Invest Pediátr Méx 2007; 10 (1): 16-20. Acceso 20 de Octubre 2015. Disponible en: http://www.medigraphic.com/pdfs/ conapeme/pm-2007/pm071d.pdf

4. Calderón M, Calle L, Hidalgo J. REPORTE DE CASO CLÍNICO: SÍNDROME DE MOEBIUS ASOCIADO AL USO DEMISOPROSTOL EN EL EMBARAZO. Rev Med FCM-UCSG 2011; 17 (1): 65-69. Acceso 20 de Octubre 2015. Disponible en: http://rmedicina.ucsg.edu.ec/ archivo/17.1/RM.17.1.09.pdf

5. Ochoa E, Pallares C, Aguinaga M, Ríos B, Ibarra J, Mayen D. SECUENCIA DE MOEBIUS: REPORTE DE CINCO CASOS Y REVISIÓN DE LITERATURA. Perinatol Reprod Hum 2008; 22 (4): 315-23. Acceso 20 de Octubre 2015. Disponible en: http://www.medigraphic.com/pdfs/ inper/ip-2008/ip084g.pdf

6. Herrera C, MendietaP,Muzzio L. REPORTE DE CASO CLÍNICO: SÍNDROME DE MOEBIUS. Rev Med FCMUCSG 2010; 16 (3): 237-42. Acceso 20 de Octubre 2015. Disponible en:http://editorial.ucsg.edu.ec/medicina/article/ view $/ 73 / 35$

7. González M. SÍNDROME DE MOEBIUS SU EXPLICACIÓN ANATÓMICA Y SU RELACIÓN EN ODONTOLOGÍA. Act Odont Venez 2010; 48 (2): 1-10. Acceso 20 de Octubre 2015. Disponible en: $h t t p: / / w w w$. actaodontologica.com/ediciones/2010/2/pdf/art20.pdf 8. Herreros MB, Rodríguez S, Franco R. EL SínDROME DE MOEBIUS: DESCRIPCIÓN DE 8 CASOS. RELACIÓN CON EL USO DE MISOPROSTOL EN EL PRIMER TRIMESTRE DEL EMBARAZO. Pediatr 2009; 36 (1): 35-41. Acceso 20 de Octubre 2015. Disponible en: http://dialnet.unirioja.es/descarga/articulo/4800220.pdf

9. Gómez L, Morales A, Cornelio R, Toledo E, Briceño M, Rivera M. ESTUDIO CLÍNICO Y GENÉTICO DEL SÍNDROME DE MOEBIUS.Bol Med Hosp Infant Mex 2008; 65: 353-357. Acceso 20 de Octubre 2015. Disponible en: http://www.medigraphic.com/pdfs/bmhim/hi-2008/ hi085d.pdf

10. Rosselli P,VillanuevaJ,AcevedoJ.PIE EQUINOVARO CONGÉNITO EN EL SÍNDROME DE MOEBIUS. Rev Col Or Tra2010; 24 (2): 112-117. Acceso 20 de Octubre 2015. Disponible en: $h t t p: / / w w w . i m b i o m e d . c o m . m x / 1 / 1 /$ articulos.php?method=showDetaile $r$ id_articulo $=66854$ \& id_ seccion $=3290$ wid_ejemplar $=6712$ wid_revista $=82$

11. Rojas V, Bustos C, Marín S,Yañez C. SÍNDROME DE MOEBIUS: DESCRIPCIÓN DE UNA SERIE DE 6 CASOS. Rev Chil Psiquiatr Neurol Infanc Adolesc 2012; 23(2): 88-92.

12. Ouanounou S, SaigalG,Birchansky S. MÖBIUS SYN-
DROME. Am J Neuroradiol 2005; 26 (2): 430-432. Acceso 20 de Octubre 2015. Disponible en: http://www.ajnr.org/ content/26/2/430.full

13. Oxley P. MOBIUS SYNDROME. En: Oxley P. Classification in facial plastic surgery. 1ra Ed. San Diego: Plural Publishing; 2009: 2-3.

14. Cammarata F. ESPECTRO CLÍNICO Y ETIOLÓGICO DEL SÍNDROME DE MOEBIUS.Arch Argent Pediatr 2007; 105 (5): 444-6. Acceso 20 de Octubre 2015. Disponible en: http://www.scielo.org.ar/scielo. php?script=sci_arttexte ${ }^{\prime}$ id $=$ S0325-00752007000500013

15. Fons MC, Póo P, Colomer J, Campistol J. SECUENCIA DE MOEBIUS. HALLAZGOS CLÍNICOS RADIOLÓGICOS. Rev Neurol 2007; 44 (10): 583-588. Acceso 20 de Octubre 2015. Disponible en: http://www. neurologia.com/pdf/Web/4410/x100583.pdf

16. Dumars S, Andrews C, Chan W, Engle E, Demer J. MAGNETIC RESONANCE IMAGING OF THE ENDOPHENOTYPE OF A NOVEL FAMILIAL MÖBIUS-LIKE SYNDROME. J AAPOS 2008 12(4): 381389. Acceso 20 de Octubre 2015. Disponible en: http:// www.ncbi.nlm.nih.gov/pmc/articles/PMC2562269/pdf/ nihms67151.pdf

17. Milet B,Alegría X. SÍNDROME DE MOEBIUS. RevObstet Ginecol 2006; 1 (3): 215-216. Acceso 20 de Octubre 2015. Disponible en: http://www.revistaobgin.cl/articulos/ ver/406

18. Sixto S, Ortega M, Aguilar M, Valdés H, Martínez R. SÍNDROME DE MOEBIUS. A PROPÓSITO DE UNA CASO. Rev Ciencias Médicas 2011; 15 (3): 215-225. Acce-

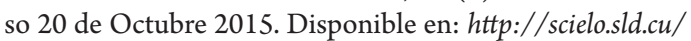
scielo.php?pid=S1561-31942011000300021 $\mathrm{\sigma}$ script $=$ sci_ arttext

19. Poveda R. SECUENCIA DE MOEBIUS EN ANESTESIOLOGÍA. Rev Chil Anest 2012; 41: 140-143. Acceso 20 de Octubre 2015. Disponible en: http://www.sachile.cl/ upfiles/revistas/50982fb0b20a7_12_poveda.pdf

20.Bos M, Hillaire D, Roux C, Faillie J,Amram D. MOBIUS SYNDROME IN A NEONATE AFTER MIFEPRISTONE AND MISOPROSTOL ELECTIVE ABORTION FAILURE. Ann Pharmacother 2008; 42 (6): 888-892.Acceso 20 de Octubre 2015. Disponible en: http://www.issues4life.org/pdfs/mobiussyndrome.pdf 21.Sánchez O, Guerra D. SINDROME DE MOEBIUS: FETOPATÍA POR MISOPROSTOL. REPORTE DE UN PACIENTE. Invest Clín 2003; 44 (2): 147-153. Acceso 20 de Octubre 2015. Disponible en: http://produccioncientifica.luz.edu.ve/index.php/investigacion/article/viewFile/10294/10283 